\title{
Study of effects of oligohydramnios on perinatal outcome
}

\author{
Aashka M. Mashkaria ${ }^{1 *}$, Babulal S. Patel ${ }^{1}$, Akshay C. Shah ${ }^{1}$, Shashwat K. Jani ${ }^{1}$, \\ Vismay B. Patel ${ }^{2}$, Adwait B. Patel ${ }^{1}$, Aastha M. Mashkaria ${ }^{1}$
}

\begin{abstract}
${ }^{1}$ Department of Obstetrics and Gynecology, Smt N. H. L. Municipal Medical College, SVPIMSR, Ahmedabad, Gujarat, India

${ }^{2}$ Department of Obstetrics and Gynecology, GCS Medical College, Hospital and Research Centre, Ahmedabad, Gujarat, India
\end{abstract}

Received: 03 April 2020

Accepted: 29 April 2020

\section{*Correspondence:}

Dr. Aashka M. Mashkaria,

E-mail: aashkamashkaria95@gmail.com

Copyright: (c) the author(s), publisher and licensee Medip Academy. This is an open-access article distributed under the terms of the Creative Commons Attribution Non-Commercial License, which permits unrestricted non-commercial use, distribution, and reproduction in any medium, provided the original work is properly cited.

\section{ABSTRACT}

Background: Oligohydramnios has got a noteworthy influence on perinatal outcome. Hence, early detection and its timely management will aid in curtailing of perinatal morbidity and mortality and leading to decreased operative interventions. Therefore, the present study is conducted to look for the effects of oligohydramnios.

Methods: This comparative study was a prospective observational study conducted at study institution. The women were divided into study and control groups based on AFI (amniotic fluid index), 100 cases were selected in each group.

Results: Out of the 200 women, included in the present study, $35 \%$ of the patients in the study group had non-reactive non-stress test (NST) while in the control group 7\% had it. Caesarean section was performed in $58 \%$ of cases in the study group as compared to $30 \%$ in the control group. Amongst these, Fetal distress was the most common indication for LSCS (lower segment caesarean section). There were no perinatal deaths in this study.

Conclusions: Based on this study it has been observed that, amniotic fluid index of $\leq 5 \mathrm{~cm}$ was commonly associated with increased LSCS rates, intrauterine growth restriction, non-reactive NST, and abnormal Doppler velocimetry studies. Therefore, every case of oligohydramnios requires to be assessed meticulously. Prompt detection; timely management and treating the underlying condition improve outcome.

Keywords: Amniotic fluid index, Doppler studies, Non-stress test, Oligohydramnios, Perinatal outcome

\section{INTRODUCTION}

The gainful outcome of obstetric wellbeing is judged by acquiring a healthy mother and healthy baby in modern obstetrics.

At term (37 weeks and beyond) the average amniotic volume is approximately $750 \mathrm{ml}$, but the volume decreases rapidly after 40 weeks. ${ }^{1}$ Oligohydramnios was defined as an amniotic fluid index (AFI) $\leq 5 \mathrm{~cm}$ and/or single deepest vertical pocket $<=2 \mathrm{~cm}$ and/or amniotic fluid volume $<=200 \mathrm{ml}$ at term. Its incidence is $1-2 \%$ of all the pregnancies. ${ }^{1}$
Liquor amnii (amniotic fluid) provides a protective milieu for the growing fetus, cushioning it against mechanical injury, maintaining temperature and allowing fetal growth and development. Clinical and radiological assessment of amniotic fluid volume as outpatient care itself is an initial step to identify high-risk pregnancy and fetal wellbeing: as variation in its amount has been related to a variety of perinatal complications. ${ }^{2}$ The amniotic fluid volume estimation is the sum of influx and efflux of fluid within the amniotic sac. Amniotic fluid volume can be measured by different methods; most commonly used being amniotic fluid index (AFI) assessment using ultrasonography. Ultrasonography is a 
non-invasive test, can be applied on a large scale as a screening method and can be used frequently. ${ }^{3}$ Amniotic fluid volume is an important component of fetal wellbeing and is part of the biophysical profile, especially in the third trimester. ${ }^{3}$ Phelan et al described amniotic fluid assessment by amniotic fluid index (AFI) through transabdominal sonography using four-quadrant techniques. ${ }^{4,5}$ A substantial decrease in AFV (amniotic fluid volume) may indicate underlying placental insufficiency, which has a definite impact on the growing fetus. Phelan and others, Baron and others and Kwon and others defined oligohydramnios as AFI less than or equal to $5 \mathrm{~cm}^{4-7}$ Oligohydramnios is caused by pregnancyinduced hypertension (PIH), congenital anomalies like renal agenesis, PROM (premature rupture of membranes), maternal dehydration (fever, vomiting, diarrhoea), drug-induced (NSAIDs), post-term pregnancies (>40 weeks), infections, idiopathic isolated, etc.

Many studies have established that oligohydramnios is associated with increased risk of gross congenital anomalies, intrauterine growth restriction, meconium aspiration syndrome, low Apgar scores, fetal distress, NICU admissions, stillbirths and increased incidence of operative interventions. ${ }^{8-10}$ Oligohydramnios is the late sign of malnutrition of the fetus. The early detection and its management will help in the reduction of perinatal morbidity and mortality at one end and decreased operative interventions at another end, ${ }^{10,11}$ Hence, the present study is undertaken to study the effect of oligohydramnios on perinatal outcome.

\section{METHODS}

The present study was a prospective observational study undertaken in the department of obstetrics and gynecology, Sardar Vallabhbhai Patel Institute of Medical and Research, Ahmedabad, Gujarat, India from February 2019 to January 2020 (12 months). Study and control group were randomly selected from women visiting in the OPD (outpatient department) and labour room.

\section{Inclusion criteria}

- At or after completed 37 weeks of gestation

- $\quad$ Singleton, intrauterine viable pregnancy

- $\quad$ AFI $<=5 \mathrm{~cm}$.

\section{Exclusion criteria}

- Congenital malformation of fetus

- Polyhydramnios (AFI>=25)

- Maternal comorbid conditions like cardiac disease, epilepsy

- Multifetal pregnancy

- Premature rupture of membranes

- Women with uterine anomalies.
The women were divided into study and control groups based on AFI. For the present study 100 cases selected in each group after fulfilling the above criteria. All women were followed up until delivery and perinatal outcome were recorded.

- Study group: AFI: $<=5 \mathrm{~cm}$

- Control group: AFI: $>5 \mathrm{~cm}$

Written and informed consent was taken from all the study participants. A detailed history and diligent clinical examination were done on admission. Baseline blood investigations, vitals, NST, daily kick count, ultrasound examination was performed on all the subjects. Clinically; fundal height and abdominal girth measurement records were kept. Phelan's four-quadrant technique by transabdominal USG was used to assess liquor Amnii volume.

If required, repeated ultrasound weekly for measuring AFI was done until the delivery. If oligohydramnios was confirmed clinically as well as by measuring AFI, regular management in the form of oral and intravenous hydration was given, the mothers were asked to keep a record of daily kick count, timely NST performed. and concurrently underlying conditions evaluated and corrected. Antepartum fetal surveillance was done as per the modified biophysical profile (BPP) and umbilical artery (UA) Doppler studies. Modified BPP includes-AFI by ultrasonography and NST.

Based on history, clinical assessment, antepartum fetal surveillance reports, blood investigations, and other obstetric factors were deciding factors for labor induction and elective/emergency caesarean section.

Patients with spontaneous onset of labor were monitored throughout and the partograph was maintained. Following the deliveries; babies were analysed for the perinatal outcomes. Maternal outcomes measured in the form of spontaneous onset/induced labor, mode of delivery, labor-related events like non-reactive NST (abnormal fetal heart rate), and indication for LSCS were studied. Perinatal outcomes in the form of Apgar score at $1 \mathrm{~min}$ and $5 \mathrm{~min}$, meconium staining, IUGR, and NICU admission were studied.

\section{RESULTS}

Table 1 is showing gestational age is compared in both groups among 200 women. Oligohydramnios was greater in 37-40 weeks of gestation.

Table 2 showing associated maternal risk factors. Placental insufficiency is the main underlying mechanism for the development of oligohydramnios in study groups. Table 3, a total $35 \%$ of the patients in the study group had non-reactive NST while in the control group $7 \%$ had nonreactive NST. 
Table 1: Gestational age in the study and control groups.

\begin{tabular}{|lcllll|}
\hline Characteristics & & Study group & Percentage & Control group & Percentage \\
\hline $\begin{array}{l}\text { Gestational age } \\
\text { (weeks) }\end{array}$ & $37-40$ & 90 & $90 \%$ & 95 & $95 \%$ \\
\cline { 2 - 6 } & $>40$ & 10 & $10 \%$ & 5 & $05 \%$ \\
\hline
\end{tabular}

Table 2: Underlying obstetrics risk factors.

\begin{tabular}{|lllll|}
\hline Risk factors & Study group & Percentage & Control group & Percentage \\
\hline Isolated oligohydramnios & 19 & $19 \%$ & - & - \\
\hline PIH (placental insufficiency) & 18 & $18 \%$ & 10 & $10 \%$ \\
\hline Severe anemia & 14 & $14 \%$ & 08 & $08 \%$ \\
\hline Hypothyroidism & 11 & $11 \%$ & 10 & $10 \%$ \\
\hline IUGR (intrauterine growth restriction) & 19 & $19 \%$ & 06 & $06 \%$ \\
\hline Fever & 03 & $03 \%$ & 02 & $02 \%$ \\
\hline Placental abruption & 05 & $05 \%$ & 01 & $01 \%$ \\
\hline
\end{tabular}

Table 3: Non-reactive NST in study and control groups.

\begin{tabular}{|c|c|c|}
\hline & Study group & Control group \\
\hline Non & $35(35 \%)$ & $07(7 \%)$ \\
\hline
\end{tabular}

Table 4: Mode of delivery in study and control groups.

\begin{tabular}{|lllll|}
\hline Mode of delivery & Study group N=100 & Percentage & Control group N=100 & Percentage \\
\hline Vaginal delivery & 042 & $42 \%$ & 070 & $70 \%$ \\
\hline LSCS & 058 & $58 \%$ & 030 & $30 \%$ \\
\hline
\end{tabular}

Table 5: Distribution of indications for caesarean section in study and control groups.

\begin{tabular}{|lllll|}
\hline Indications for LSCS & Study group $\mathbf{n = 5 8}$ & Percentage & Control group $\mathbf{n = 3 0}$ & Percentage \\
\hline Fetal distress & 38 & $65.5 \%$ & 21 & $\mathbf{7 0 \%}$ \\
\hline Malpresentation & 01 & $1.7 \%$ & 01 & $3.33 \%$ \\
\hline Induction failure & 05 & $8.62 \%$ & 02 & $6.66 \%$ \\
\hline Non-progress of labor & 03 & $5.17 \%$ & 05 & $16.66 \%$ \\
\hline Cephalo pelvic disproportion & 01 & $1.7 \%$ & 01 & $3.33 \%$ \\
\hline Abnormal Doppler changes & 11 & $18.96 \%$ & - & - \\
\hline
\end{tabular}

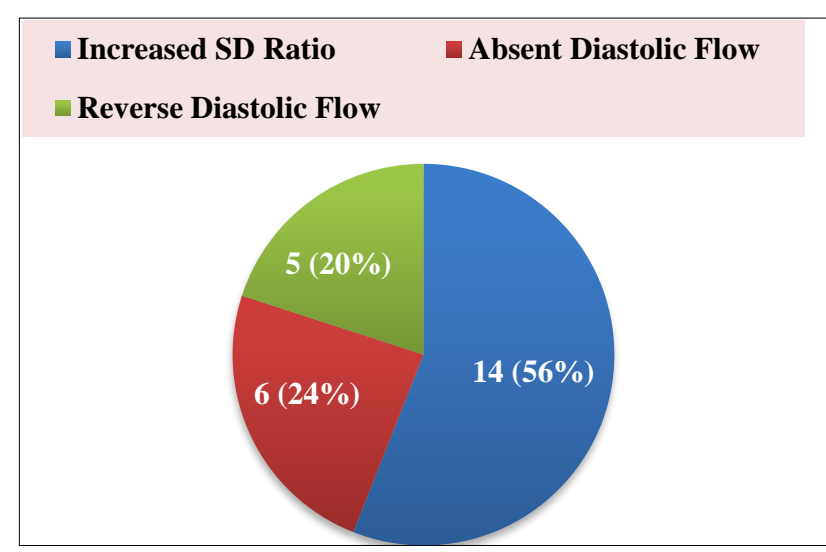

Figure 1: Doppler changes in study group $(\mathrm{n}=\mathbf{2 5})$.

Table 4, this is showing mode of delivery in study and control groups. Caesarean section was required in $58 \%$ of cases in the study group as compared to $30 \%$ in the control group.

This shows the distribution of indications for the caesarean section in the study and control groups. Fetal distress was the most common indication for LSCS in both groups. In the study group, the second most common cause of LSCS was Altered Doppler such as reversal of the umbilical artery flow or absent diastolic umbilical artery flow.

Figure 1 showing Doppler abnormalities in the study group, 25 subjects had doppler abnormality. Increased SD ratio was observed most commonly. Table 6, there were no perinatal deaths in this study. Among neonatal outcomes, induction of labor, meconium stained liquor aspiration, non-reactive NST, and Apgar score <7 at 1 min in the study group were statistically significant. 
Table 6: Fetal outcome.

\begin{tabular}{|lllll|}
\hline Fetal outcome & Study group, $\mathbf{N}=\mathbf{1 0 0}$ & Percentage & Control group, N=100 & Percentage \\
\hline IUGR & 033 & $33 \%$ & 006 & $06 \%$ \\
\hline Apgar <7 at 1 min & 035 & $35 \%$ & 015 & $15 \%$ \\
\hline Apgar < at 5 min & 020 & $20 \%$ & 007 & $07 \%$ \\
\hline Non-reactive NST & 036 & $36 \%$ & 010 & $10 \%$ \\
\hline $\begin{array}{l}\text { Meconium stained liquor } \\
\text { aspiration }\end{array}$ & 030 & $30 \%$ & 016 & $16 \%$ \\
\hline NICU admission & 035 & $35 \%$ & 020 & $20 \%$ \\
\hline
\end{tabular}

\section{DISCUSSION}

Same to this present study, many studies have proven that oligohydramnios is well-correlated with increased risk of perinatal morbidity and mortality. Regular assessment of amniotic fluid volume is a necessary part of antenatal fetal surveillance. ${ }^{11}$ As shown in Table 1, the incidence of oligohydramnios observed is greater in 37-40 weeks of gestation due to various reasons in this present study. Medical disorders like preeclampsia were risk factors to cause oligohydramnios in this study. Chronic placental insufficiency is the main underlying mechanism, leading to oligohydramnios. In the present study, preeclampsia was seen in $18 \%$ of cases in the study group and $10 \%$ of cases in the control group. In Chate $\mathrm{P}$ et al study hypertensive disorders found in $8 \%$ of oligohydramnios cases only. ${ }^{12}$ Abruptio placenta was observed in 5\% of the present study and a similar incidence was reported by Chandra $\mathrm{P}$ et al (7.69\%). ${ }^{13}$ Hypothyroidism was seen at the same incidence in both study and control groups in the present study turned out to be insignificant. Induction of labor was seen in $28 \%$ of women with low AFI in the study group as compared to the control group $12 \%$. Singhal SR et al study significantly higher induction of labor was seen in low AFI group $72 \%$ as compared to the control group $12 \% .^{14}$

The most common indication for LSCS was fetal distress followed by ultrasound Doppler abnormalities and then failed induction in the study group. While in the control group fetal distress followed by non-progress of labor. In the present study, study group underwent LSCS in 58\% of cases, while in the control group 30\% undergone LSCS. Similar to Mathuriya et al study $65 \%$ of the study cases and only $10 \%$ of controls underwent LSCS. ${ }^{15} 90 \%$ of the controls were delivered vaginally in both studies. In Ranjita $\mathrm{G}$ et al, study caesarean section rate was $54 \%$ in the oligohydramnios group as compared to the control group $26 \% .^{16}$

Table 7: Perinatal outcome in different studies.

\begin{tabular}{|lllllllll|}
\hline \multirow{2}{*}{ Studies } & Non-reactive NST & \multicolumn{2}{c|}{ NICU admission } & IUGR & \multicolumn{2}{c|}{ Meconium stained liquor } \\
\hline Ranjita G et al & Study & Control & Study & Control & Study & Control & Study & Control \\
\hline Bhagat et al & $32 \%$ & $15 \%$ & $28 \%$ & $18 \%$ & $62 \%$ & $25 \%$ & $18 \%$ & $12 \%$ \\
\hline Bachhav AA et al ${ }^{17}$ & $65 \%$ & $24 \%$ & $36 \%$ & $24 \%$ & $56 \%$ & $21.7 \%$ & $16 \%$ & $14.9 \%$ \\
\hline Present study & $35 \%$ & $07 \%$ & $33 \%$ & $10 \%$ & $30 \%$ & $11 \%$ & $18 \%$ & $01 \%$ \\
\hline
\end{tabular}

Controversies encountered in relation to oligohydramnios and meconium-stained liquor. In the present study, induction of labor, non-reactive NST, meconium-stained liquor aspiration, IUGR, $1 \mathrm{~min}$ Apgar score and $<7 \mathrm{~min}$ Apgar score were statistically significant and meticulous differences found between study and control groups.

Outcomes like meconium-stained liquor, 1-min Apgar score <7, IUGR and admission to NICU were not statistically different in the oligohydramnios group from the normal group in Ranjita et al study. ${ }^{16}$ Table 7, different studies are compared on the basis of these parameters-IUGR, meconium-stained liquor, non-reactive
NST, NICU admissions. Non-reactive NST and meconium stained liquor rates were higher in the study group in all the studies. IUGR babies and babies requiring the NICU admission rates were higher in the study group in all the studies, including the present study.

According to statistics; the screening by AFI turns out to be a very nice noteworthy screening test.

Variations in incidences of perinatal and maternal outcomes could be because of differences in the patient selection criteria, the selection of cases for the study. 


\section{ACKNOWLEDGMENTS}

Authors would like to thank the department of obstetrics and gynecology and department of radiology, Sardar Vallabhbhai Patel Institute of Medical Science and Research. Authors would also like to thank the participant mothers.

Funding: No funding sources

Conflict of interest: None declared

Ethical approval: The study was approved by the Institutional Ethics Committee

\section{REFERENCES}

1. Manning FA. Antepartum fetal testing: a critical appraisal. Curr Opin Obstet Gynecol. 2009;21(4):348-52.

2. Chamberlain PF, Manning FA, Morrison I. The relationship of marginal and decreased amniotic fluid volumes to perinatal outcome. Am J Obstet Gynecol. 1984;150(3):245-9.

3. Bhagat M, Chawla I. correlation of amniotic fluid index with perinatal outcome. Ind J Obstet Gynecol. 2014;64(1):32-5.

4. Phelan JP, Smith CV, Broussard P, Small M. Amniotic fluid volume assessment with the fourquadrant technique at $36-42$ weeks gestation. J Reprod Med. 1987;32(7):540-2.

5. Rutherford SE, Phelan JP, Smith CV, Jacobs N. The four-quadrant assessment of amniotic fluid volume: an adjunct to antepartum fetal heart rate testing. Obstet Gynecol. 1987;70:353-6.

6. Baron C, Morgan MA, Garite TJ. The amniotic fluid volume assessed intrapartum on perinatal outcome. Am J Obstet Gynecol. 1995;173:167-74.

7. Kwon JY, Kwon HS, Kim YH, Park YW. Abnormal Doppler velocimetry is related to adverse pregnancy outcomes for the borderline amniotic fluid index in the third trimester. J Obstet Gynecol Res. 2006;32:545-9.
8. Nath J, Jain M, Najam R. A clinical study on oligohydramnios in the third trimester of pregnancy with special emphasis on the perinatal outcome. J Evol Med Dental Sci. 2013;2(39):7386-91.

9. Bangal VB, Giri PA, Sali BM. Incidence of oligohydramnios during pregnancy and its effects on maternal and perinatal outcomes. JPBMS. 2011;12(5):1-4.

10. Bachhav AA, Manjushri W. Low amniotic fluid index at term as a predictor of adverse perinatal outcome. J Obstet Gynecol India. 2014;64(2):120-3.

11. Ott WJ. Current perspective in antenatally surveillance ultrasound. Rev Obst Gynaecol. 2003;3:1-180.

12. Chate $P$, Khatri M, Hariharan C. Pregnancy outcome after diagnosis of oligohydramnios at term. Int $\mathbf{J}$ Reprod Contracept Obstet Gynecol. 2013;2:23-6.

13. Chandra P, Kaur SP, Hans DK, Kapila AK. The impact of amniotic fluid volume assessed intrapartum on perinatal outcomes. Obstet Gynae Today. 2000;5(8):478-81.

14. Singhal SR, Gupta R, Sen J. Low amniotic fluid index as a predictor of adverse perinatal outcome: an Indian perspective. Clinics Mother Child Health. 2015;12:201.

15. Mathuriya G, Verma M, Rajpoot S. Comparative study of maternal and fetal outcome between low and normal amniotic fluid index at term. Int J Reprod Contracept Obstet Gynecol. 2017;6:640-4.

16. Ranjita G, Sandhyarani B. Correlation of Oligohydramnios with Perinatal Outcome. J Med Res Prac. 2017;6(3):93-5.

17. Bachhav AA, Manjushri W.Low amniotic fluid index at term as a predictor of adverse perinatal outcome. $\mathrm{J}$ Obstet Gynecol India.2014;649)-10-3.

Cite this article as: Mashkaria AM, Patel BS, Shah AC, Jani SK, Patel VB, Patel AB, et al. Study of effects of oligohydramnios on perinatal outcome. Int J Reprod Contracept Obstet Gynecol 2020;9:2525-9. 